\title{
EFECTO DE LA HUMEDAD EN EL ALMACENAMIENTO HERMÉTICO A CORTO PLAZO DE SEMILLAS DE FRIJOL (Phaseolus vulgaris)1
}

\author{
Roberto Aguirre ${ }^{2}$
}

\begin{abstract}
COMPENDIO
Se almacenaron semillas de frijol (Phaseolus vulgaris) variedad Calima, durante 32 semanas en empaques herméticos, a $30^{\circ} \mathrm{C}$ con siete niveles de humedad comprendidos entre 10.2 y $143 \%$, La calidad física y fisiológica de la semilla fue evaluada cada dos semanas mediante muestreos a lotes Independientes de cada uno de los niveles de humedad. Con los datos de germinación y brotación contratiempo de almacenamiento, se desarrolló un modelo matemático con coeficientes de determinación (r2) de 0.85 y 0.93 , respectivamente. Los resultados obtenidos permiten concluir que la semilla de frijol variedad Calima con alta calidad Inicial y con una humedad máxima M $12 \%$, al ser almacenada herméticamente en un ambiente a $30^{\circ} \mathrm{C}$ hasta por ocho meses no sufre pérdidas significativas en su calidad fisiológica.
\end{abstract}

Palabras clave adicionales: Calidad semilla.

\section{INTRODUCCIÓN}

El frijol (Phaseolus vulgaris) es un cultivo de subsistencia de gran importancia socioeconómica, especialmente en los países de América Latina y Africa, donde en 1986 se produjeron más de 6.2 millones de toneladas de este grano, en 10.9 millones de hectáreas, con un rendimiento promedio de $569 \mathrm{~kg} / \mathrm{ha}$ (CIAT, 1988b). En América Latina, el 77\% M frijoles producido por pequeños agricultores en parcelas cuyo tamaño medio es de 3.3 ha (FAO, 1987). Estos agricultores cultivan el frijol como grano para el consumo de su familia, llevan al mercado el excedente si lo hay, y separan una cantidad determinada, la cual almacenan para utilizarla en la siguiente siembra. En Colombia, por ejemplo, menos del $1 \%$ de la semilla de frijol sembrada en 1986 era semilla comercial (ICA, 1987). Se ha encontrado que aunque en muchos casos la calidad del frijol cosechado es buena, debido al manejo intensivo que los pequeños agricultores dan a sus lotes de frijol, las malas condiciones de manejo y almacenamiento posteriores a la cosecha reducen esta calidad, haciendo que se reduzca también considerablemente el potencia; de la semilla empleada.

Es prudente enfatizar aquí que unas buenas condiciones de almacenamiento no pueden compensar o corregir el deterioro causado por una cosecha tardía e inapropiada, el daño mecánico, o el secamiento inadecuado; el deterioro causado por las anteriores con-

\begin{abstract}
${ }^{3}$
Bean seed variety "Calima" (Phaseolus vulgaris) was stored for 32 weeks in hermetic containers at $30^{\circ} \mathrm{C}$ using seven different moisture levels within 10.2 and $14.3 \%$. Seed quality was evaluated every two weeks by sub-sampling independent lots from each controlled moisture level. Linear models of germination and budding versus storage time had $\mathrm{r}^{2}$ values of 0.85 and 0.93 respectively. These results indicate that "Calima" seed with high Initial quality and grain moisture less than $12 \%$ can be stored in hermetic containers for up to 8 months at temperatures of $30^{\circ} \mathrm{C}$ with little loss of physiologycal quality.
\end{abstract}

diciones puede continuar aunque las semillas se almacenan en condiciones ideales (Bass, 1973). Semillas maduradas, cosechadas y beneficiadas en diferentes circunstancias estarán sujetas a diferentes niveles de deterioro y tendrán diferente calidad (Roberts, 1972).

Sin restarle importancia a los factores mencionados anteriormente, es esencial conservar la calidad inicial de la semilla, mediante prácticas adecuadas de almacenamiento, si se espera obtener una buena productividad del cultivo. Las principales causas de problemas durante el almacenamiento se deben a la utilización de semillas de baja calidad, semillas con un alto contenido de humedad $(\mathrm{H})$; períodos de almacenamiento muy largos, y almacenes húmedos, calientes, poco ventilados y con condiciones sanitarias inadecuadas (Delouche, 1973).

Si el material que el pequeño productor va a utilizar como semilla proviene del grano obtenido de su propia cosecha anterior, o de la de su vecino, debe almacenarlo durante 2 ó 3 meses (si hace dos campañas por año) o durante 8 meses (en el caso de una campaña anual). Así, aunque la calidad inicialdel material sea buena, las condiciones de almacenamiento deficientes contribuyen decisivamente a acelerar el proceso de deterioro y, consecuentemente, a la rápida pérdida de la

\footnotetext{
${ }^{1}$ Presentado en la XXXV Reunión Anual del PCCMCA, San Pedro Sula, Honduras. 1989.

2 Unidad de Semillas, CIAT, Colombia.

${ }^{3} \mathrm{El}$ abstract es traducción del compendio. Elaborado por el Comité Editorial para mostrar el formato de presentación de los artículos.

${ }^{4}$ Publicado en Agronomía Mesoamericana, Vol. 1 (1990)
} 
calidad fisiológica de las semillas.

La calidad fisiológica de las semillas incide directamente en la emergencia, en el establecimiento y en el rendimiento del cultivo. Estudios llevados a cabo para determinar la incidencia de la calidad fisiológica de la semilla en el rendimiento de los cultivos han encontrado que la baja calidad de la semilla puede resultar en disminuciones hasta del $10 \%$ en la productividad del cultivo (Delouche, 1985).

En América Latina, las pérdidas han promediado el $15 \%$ de la producción, pero pueden alcanzar hasta el 35\% (México y América Central) (CIAT, 1988a). Estas altas pérdidas se deben principalmente al ataque de insectos (bruchidos o gorgojos) durante el almacenamiento, entre los cuales se destacan Acanthoscelides obtectus (Say) en climas templados y Zabrotes subfasciatus (Boheman) en climas cálidos. Las condiciones óptimas para el desarrollo de estos insectos se dan a una temperatura (T) de 30'C y a una humedad relativa (HR) del 70\%, prevalecientes en gran parte del trópico (Schwartz y Gálvez, 1980).

Por estas razones, es de suma importancia para el pequeño agricultor no sólo producir semillas de buena calidad, sino también mantener esa calidad hasta la siguiente siembra, mediante la utilización de sistemas de almacenamiento adecuados.

Un método muy antiguo, utilizado empíricamente en muchas regiones para el almacenamiento de granos y semillas, es el almacenamiento hermético $(\mathrm{AH})$, especialmente el almacenamiento subterráneo, sobre el cual se han reportado estudios desde 1819 (Sigaut, 1980). Para utilizar este sistema de almacenamiento es absolutamente esencial secar previamente las semillas hasta un nivel de $\mathrm{H}$ seguro (dependiendo de la $\mathrm{T}$ máxima de almacenamiento) antes de sellar herméticamente el recipiente, puesto que si la semilla tiene una $\mathrm{H}$ alta, el $\mathrm{AH}$ causará más daño a las semillas que el almacenamiento abierto (Harrington, 1959).

Es posible guardar semillas secas en $\mathrm{AH}$ durante 2 ó 3 años a temperaturas ordinarias ( 21 a $32 \mathrm{~T}$ ) y durante mucho más tiempo a menores temperaturas. Por ejemplo, los bancos de germoplasma almacenan semillas durante períodos de más de 20 años, con bajos contenidos de $\mathrm{H}, 5 \%$ base húmeda (bh), en empaques herméticos a muy bajas temperaturas (-20T).

Desafortunadamente, hasta ahora no se han hecho muchos estudios concienzudos sobre el AH como alternativa para mantener la calidad de pequeñas cantidades de semillas durante períodos cortos de tiempo. Esta posibilidad podría. llegar a tener un amplio rango de utilización, especialmente en regiones tropicales, donde la escasez de recursos limitan el acceso a instalaciones y equipos para controlar la T y/o HR del ambiente de almacenamiento.

$\mathrm{El} \mathrm{AH}$ tiene varias ventajas que pueden llegar a ser atractivas para el pequeño agricultor-, a) Cuando la se- milla se almacena con la $\mathrm{H}$ apropiada, la calidad de las semillas se mantiene durante más tiempo que cuando se almacena en ambientes abiertos; b) Debido a las características de hermeticidad del recipiente, la $\mathrm{H}$ de la semilla no se ve afectada por la HR del ambiente en el exterior del recipiente; e) La HR dentro de los empaques herméticos fluctúa muy poco y sólo debido 1 a los cambios de T; d) Si la $\mathrm{H}$ de la semilla es lo suficientemente baja, el efecto de la $\mathrm{T}$ en la calidad de la semilla se reduce, haciendo innecesario el acondicionamiento de la $\mathrm{T}$ de la bodega; e) El recipiente actúa no sólo como barrera; c vapor, sino también como protección contra roedores, hongos, insectos y riesgos de contaminación.

Considerando la importancia de desarrollar una tecnología sencilla que permitiera al pequeño agricultor, a nivel individual o en grupo, utilizar el método de AH en forma práctica, se decidió realizar una investigación en la Unidad de Semillas del CIAT en Cali, Colombia, para determinar cuál era el nivel de $\mathrm{H}$ seguro para almacenar semilla de frijol herméticamente en climas cálidos y/o húmedos, durante un período suficien para garantizar semilla de buena calidad, al momento de la siguiente siempre.

El objetivo específico del estudio consistió en determinar el efecto de la humedad en la calidad de la semilla de frijol, variedad Calima, cuando ésta se almacena herméticamente durante 32 semanas a una temperatura de $30^{\circ} \mathrm{C}$.

\section{REVISION DE BIBLIOGRAFIA}

Es ampliamente aceptado que los factores mas importantes en el almacenamiento de semilla, aparte de su calidad inicial, son su contenido de humedad y la temperatura),1 concentración de oxígeno del ambiente en que se almacena (Roberts, 1972). Entre estos, la alta humedad es la causa principal de la pérdida de viabilidad de la semilla.

Por otro lado, los factores ambientales que inciden directamente, en la $\mathrm{H}$ de las semillas son la $\mathrm{T}$ y la HR del aire en contacto con ellas, ya que las semillas y el aire son materiales higroscópicos y tienden a ganar o a perder humedad, dependiendo del ambiente en que se encuentren, hasta alcanzar lo que se conoce como contenido de humedad en equilibrio ( $\mathrm{CHE}$ ).

En regiones cálidas y húmedas, la mayoría de semillas disminuyen sustancialmente su viabilidad y su vigor en un período de sólo seis meses, cuando son guardadas en almacenamiento abierto (AA), a $30{ }^{\circ} \mathrm{C}$ y $75 \%$ HR (Delouche1980). Para almacenamiento a corto plazo, la práctica ha demostrado que es suficiente controlar sólo uno de esos factores (T o HR).

Se ha estimado que es posible mantener la viabilidad de la semilla de frijol hasta por un año en AA a $27^{\circ} \mathrm{C}$, 
si la $\mathrm{H}$ de las semillas es menor del $8 \%$ (Harty, 1977). Otro problema que se presenta en el almacenamiento de semillas de frijol es el ataque de insectos, especialmente gorgojos, los cuales sólo dejan de ser activos en semillas con una $\mathrm{H}$ inferior al $8 \%$ (Roberts, 1972). Pero pueden sobrevivir en condiciones de muy bajas concentraciones de oxígeno (Burrell, 1980). Por tanto, los gorgojos estarán activos en condiciones de $\mathrm{AH}$, independientemente de la HR y de hermeticidad del recipiente. Esto hace necesario controlar 1os insectos antes de colocar las semillas en AH. Existen algunos métodos ,sencillos que ayudan a controlar la infestación de gorgojos durante el almacenamiento. Los agricultores tradicionalmente han utilizado ceniza, pimienta o restos de cosecha, los cuales mezclan con las semillas. Un buen control se logra aplicando $5 \mathrm{ml} \mathrm{de}$ aceite por cada kilogramo de semilla que se va a almacenar. También es efectivo el uso de productos químicos, tales como los Piretrinas o la Fosfamida, las cuales controlan los gorgojos y tienen muy bajo efecto residual (Schwartz y Gálvez, 1980).

No existe mucha información sobre las condiciones apropiadas de $\mathrm{H}$ y $\mathrm{T}$ que permitan el $\mathrm{AH}$ de semillas de frijol a corto plazo, pues se ha dado mayor énfasis al almacena miento a largo plazo. Se ha recomendado que para el $\mathrm{AH}$ de semillas, su $\mathrm{H}$ no debe ser superior al 5\% y para el caso de frijol el límite es del 9\% (Harrington, 1959; Harrington y Douglas, 1970; Delouche, 1973; Base, 1975; Justice y Bass, 1978). Se han almacenado semillas de frijol con un $9 \%$ de $\mathrm{H}$, en buenas condiciones durante 15 años, en recipientes herméticos a temperatura ambiente (Nakamura, 1975). En general se considera que el rango de $\mathrm{H}$ apropiado para el AH es del 6 al 12\% (Harrington, 1973).

Sin embargo, hay alguna evidencia que indica que es posible almacenar semillas de frijol con "alta" $\mathrm{H}$ en ambientes con $\mathrm{T}$ alta durante cortos períodos de tiempo. Se han hecho ensayos de $\mathrm{AH}$ de semillas de frijol con $12.7 \%$ de $\mathrm{H}$ hasta 12 meses a $19^{\circ} \mathrm{C}$; en estas condiciones, la germinación de las semillas disminuyó de 90 a $84 \%$; y semillas con $10.9 \%$ de $\mathrm{H}$ almacenadas a $23^{\circ} \mathrm{C}$, que disminuyeron su germinación de 95 a 82\%(Martínez, 1986). Se ha reportado que semillas de frijol almacenadas durante 250 días a $27^{\circ} \mathrm{C}$ en ambientes con una HR del 44,66 y 78\% disminuyeron su germinación en $97 \%$ a 92,87 y $0 \%$, respectivamente.

Asimismo, se ha encontrado que semillas de frijol almacenadas a $30^{\circ} \mathrm{C}$ en AA y en $\mathrm{H}$ perdieron rápidamente su calidad en ambos casos (Justice y Bass, 1978). Akamine, citado por Roos (1988), reporta el caso de semillas de frijol con una germinación inicial del 92\%, almacenadas durante 12 meses en un rango de 21 a $27^{\prime} \mathrm{C}$, las cuales presentaron una germinación del $76 \%$ al ser almacenadas a $45 \%$ HR y del $88 \%$ cuando se almacenaron a $60 \%$ HR.

Wilson (1986) presenta datos de AH de semillas de frijol, variedad Calima, en un amplio rango de tempe- raturas $(-15$ a $60 \mathrm{~T})$ y de contenidos de humedad $(8 \mathrm{a}$ $24 \%$ ). Entre otros, presenta datos de semilla con $8 \%$ de $\mathrm{H}$ almacenada a $32^{\circ} \mathrm{C}$, que luego de 216 días presenta una disminución en su germinación de 94 a 93\%; semillas con $12 \%$ de $\mathrm{H}$ almacenada a $32 \mathrm{~T}$, que a los 216 días presenta una disminución de germinación desde $95 \%$ hasta 90 y $0 \%$ a los 30 y 108 días, respectivamente. También presenta datos de lectura temprana de germinación de 95 a 94\%; y semillas con $16 \%$ de $\mathrm{H}$, almacenadas a $32^{\circ} \mathrm{C}$, que presentaron disminuciones en su germinación (4 días), los cuales pueden ser interpretados corno pruebas de vigor. Las semillas con $8 \%$ de $\mathrm{H}$ almacenadas a $32^{\circ} \mathrm{C}$, presentaron una disminución en el vigor desde 39 hasta $4 \%$ luego de 216 días; las semillas con $12 \%$ de $\mathrm{H}$ almacenadas a $32^{\prime} \mathrm{C}$, disminuyeron su vigor desde 73 hasta $10 \%$; y las semillas con $16 \%$ de $\mathrm{H}$ almacenadas a la misma $\mathrm{T}$, presentaron una pérdida total de vigor luego de 216 días.

De esta información se puede intuir que $16 \%$ es una $\mathrm{H}$ muy alta y que $8 \%$ es muy baja para $\mathrm{AH}$ a altas $\mathrm{T}$ y a corto plazo; debe existir un valor de $\mathrm{H}$ "seguro" ubicado entre estos dos límites y muy cercano al $12 \%$ de H. Por esta razón, se decidió estudiar más de cerca el efecto de la $\mathrm{H}$ en el rango entre 10 y $14 \%$; el tiempo de almacenamiento se fijó en ocho meses que es el período máximo requerido para el caso en que se hace una sola campaña por año-, y se escogió una $\mathrm{T}$ igual a $30^{\circ} \mathrm{C}$, la cual representa la máxima temperatura ambiente promedio a la cual se almacena semilla de frijol en climas tropicales.

\section{METODOLOGIA}

\section{A. Metodología General}

Se almacenaron semillas certificadas de fríjol, variedad Calima, provenientes de un lote comprado a un productor de semillas de] Valle del Cauca, con un contenido de humedad inicial del $14.3 \%$ (bh). Se decidió utilizar semilla certificada, ya que el material producido por los agricultores es muy heterogéneo y se buscaba diminuir posibles efectos causados por esa diversidad en la calidad genética de la semilla. Cada kilogramo de semilla fue tratado con 6 ppm de clorpirifos-metil al $43.2 \%$ (Reldan 4E) y $0.5 \mathrm{~g}$ de fosfuro de aluminio al 57\% (Detia-gas), con el fin de controlar los insectos antes de empacar las semillas.

Se almacenaron semillas con siete contenidos de $\mathrm{H}$ diferentes, comprendidos entre $14.3 \%$ ( $\mathrm{H}$ inicial de la semilla) y $10.2 \%$. Las semillas se almacenaron durante 32 semanas y cada dos semanas se tomaron muestras; por tanto, se utilizaron 17 muestras (incluyendo la muestra inicial) de cada uno de los siete niveles de $\mathrm{H}$, para un total de 119 muestras.

Las 17 muestras correspondientes al mayor contenido de $\mathrm{H}$ (14.3\%) fueron obtenidas de la semilla recibida, una vez fumigada y homogeneizada. Las se- 
millas restantes fueron colocadas en un secador estacionario de capa delgada $(10 \mathrm{~cm})$, en contacto con airea 30 'C. La $\mathrm{H}$ de las semillas se determinó a intervalos frecuentes; cuando se alcanzaba el nivel de $\mathrm{H}$ deseado, se procedía a homogeneizar la semilla ya obtenerlas 17 muestras correspondientes a ese nivel. La semilla restante continuaba en el secador hasta alcanzar el siguiente nivel de $\mathrm{H}$; el procedimiento se repitió hasta obtener muestras para los siete niveles de humedad.

Se evaluó la calidad de cada una de las siete muestras iniciales mediante determinación de humedad (ISTA, 1985), germinación (ISTA, 1985) y emergencia en el campo (Popinigis, 1974).

Las 112 muestras restantes fueron colocadas en sendos recipientes plásticos. Se utilizaron tarros plásticos cilíndricos de $20 \mathrm{~cm}$ de altura y $10 \mathrm{~cm}$ de diámetro, con paredes de $0.75 \mathrm{~mm}$ de espesor, un volumen interior de 1200 $\mathrm{cm}^{3}$, y un peso de $100 \mathrm{~g}$. Tan pronto como la semilla era colocada en los recipientes, se cerraban con tapa a presión y contratapa roscada, ambas del mismo material plástico. Con el fin de asegurar la hermeticidad de los tarros, estos fueron sellados adicionalmente con cinta plástica.

Una vez sellados, se pesó cada recipiente y se colocó en una cámara de almacenamiento, la cual se mantuvo a una $\mathrm{T}$ de $30^{\circ} \mathrm{C}$ durante los ocho meses que duró el ensayo. Los recipientes, completamente llenos con semilla, cerrados y sellados, pesaron aproximadamente $950 \mathrm{~g}$ de cada uno.

Cada dos semanas se retiraba de la cámara de almacenamiento una muestra de cada uno de los niveles de $\mathrm{H}$, para un total de siete muestras. Luego que eran removidos de la cámara, se examinaba el sellado de los recipientes y se determinaba si había habido algún cambio en el peso.

Posteriormente se abría el tarro, se introducía un sensor, y se cerraba rápidamente. Este sensor indicaba la $\mathrm{T}$ y la HR M aire ambiente interior en contacto con las semillas, con el fin de posteriormente relacionar estas dos mediciones con la $\mathrm{H}$ de la semilla en equilibrio en esas condiciones. Tan pronto se estabilizaba la semilla, y se tomaba una muestra para determinar su contenido de humedad.

La semilla restante era examinada con el fin de detectar daños en su apariencia y luego era colocada en un cuarto a $17^{\circ} \mathrm{C}$ y $50 \% \mathrm{HR}$ donde permanecía durante dos días, con el fin de uniformizar la humedad de los diferentes lotes antes de proceder a tomar las muestras para las pruebas de germinación. De esta forma se buscaba minimizar el efecto que la diferencia de humedades pudiera tener en los resultados de las pruebas de germinación. Las semillas restantes permanecían en este cuarto hasta el final del ensayo. En ese momento, se retiraron todas las muestras y se hizo la prueba de emergencia en el campo.

\section{B. Evaluación de la Calidad de las Semillas}

\section{Calidad Física}

Se realizaron una serie de mediciones con el fin de evaluar las siguientes características físicas de la semilla y del ambiente de almacenamiento:

a. Pérdida de peso: El recipiente, lleno de semillas, cerrado y sellado se pesó al inició y al final del almacenamiento para determinar cambios de peso durante ese período. Se utilizó una balanza digital de $0.01 \mathrm{~g}$ de resolución y $1600 \mathrm{~g}$ de capacidad.

b. Humedad de la semilla: Se determinó la $\mathrm{H}$ de la semilla en tres replicaciones, moliéndola en un molino especial y secándola en la estufa durante 1 hora a $130^{\circ} \mathrm{C}$, de acuerdo con los procedimientos internacionalmente establecidos (ISTA, 1985).

c. Temperatura y humedad relativa del aire: Se midió la $\mathrm{T}$ y HR del aire dentro del recipiente al final del almacenamiento y se relacionó con el CRE de las semillas. Se utilizó un termohigrómetro marca Hygrotest modelo 6400. Con el fin de no alterar las condiciones en el interior del recipiente, se introducía rápidamente el sensor y de nuevo se sellaba la tapa hasta que la lectura del instrumento se hubiera estabilizado. Estas mediciones no se pudieron hacer para todas las muestras, pues el instrumento $\mathrm{n}$ estuvo disponible desde el inicio $\mathrm{M}$ ensayo.

d. Temperatura del cuarto de almacenamiento: La temperatura de la cámara de almacenamiento se midió permanentemente mediante un termógrafo y se controló con u termostato con el fin de garantizar que se mantuviera en( nivel deseado $\left(30^{\circ} \mathrm{C}\right)$.

e. Apariencia: Al retirar cada recipiente de la cámara almacenamiento, se revisaba el estado del recipiente, especialmente el sistema de cerramiento; una vez abierto, se observaba la apariencia física de la semilla y el posible daño causado por insectos.

\section{Calidad Fisiológica}

Se realizaron pruebas de campo y de laboratorio con fin de evaluar la calidad fisiológica de las semillas. Las pruebas fueron realizadas por el personal de Laboratorio de Control de Calidad de la Unidad de Semillas del CIAT.

a. Germinación: La semilla se puso a germinar en arena; hicieron cuatro replicaciones de 50 semillas cada una, con un único conteo a los siete días. En estas pruebas se utilizó arena esterilizada con $25 \%$ de humedad, de acuerdo con las normas internacionales (ISTA, 1985).

b. Emergencia: Al finalizar el ensayo se sembraron en campo simultáneamente 3 replicaciones de 100 
semillas cada una de todas las muestras. La lectura de emergencia se hizo a los 15 días (Popinigis, 1974).

c. Análisis estadístico

\section{Ecuación de Viabilidad}

El análisis estadístico realizado con los datos de germinación y emergencia obtenidos cada dos semanas durante las 32 semanas de almacenamiento, consistió en obtener modelo matemático que simulara los datos experimentales observados, con el fin de generar curvas de cambios en la germinación y la emergencia de las semillas, en función de su contenido de $\mathrm{H}$ y del período de almacenamiento.

Para lograr este objetivo se ensayaron diferentes modelos matemáticos de superficie de respuesta, con el fin de determinar los coeficientes de la ecuación que mejor simulara los datos experimentales. La variable dependiente considerada es la viabilidad de las semillas (V), la cual puede e representada por el porcentaje de germinación $(\mathrm{G})$, o por el porcentaje de emergencia (E) de las semillas. Las variables independientes consideradas fueron el contenido de humo de las semillas (\%bh) y el tiempo (S) de almacenamiento en semanas. La temperatura de las semillas durante el almacenamiento no fue tenida en cuenta para el análisis, pues se mantuvo constante a $30^{\circ} \mathrm{C}$ para todos los tratamientos.

Los modelos matemáticos probados fueron los siguientes: Un modelo polinómico de segundo grado; la ecuación de viabilidad de Ellis y Roberts (Roberts, 1961, 1972; Ellis y Roberts, 1980; Wi1son, 1986; Ellis, 1988); el modelo propuesto por Gompertz (Berger, 1981); y el modelo de Houston (Houston, 1973). Este último transforma la variable dependiente viabilidad (V), a valores "logit" de viabilidad (Lv) utilizando el logaritmo natural (Ln):

$$
\mathrm{Ly}=[\mathrm{Ln}(100-\mathrm{V}) / \mathrm{V}]
$$

Todos estos modelos fueron ensayados con relativo éxito, pero el que mejores resultados dio fue la siguiente versión modificada del modelo de Houston, basado en la transformación logit:

$$
\mathrm{Ly}=\mathrm{a}+\mathrm{b} \cdot \mathrm{S}^{2} \cdot \exp (\mathrm{H})
$$

Donde $\underline{\mathrm{S}}$ es tiempo de almacenamiento,; $\mathrm{H}$ es humedad de la semilla; $\underline{a}, \underline{b}$, son los coeficientes que se quieren determinar; y exp es el operador exponencial, base de los logaritmos naturales. Los coeficientes a y $\underline{b}$ fueron determinados por el método de regresión lineal, utilizando el paquete estadístico SAS de la Unidad de Servicio de Datos del CIAT. Se uso el término Lv como variable dependiente y $\mathrm{S}^{2}, \exp (\mathrm{H})$ como variable independiente. Una vez obtenidos estos valores, fue posible calcular directamente al valor de V mediante la siguiente expresión, obtenida de la ecuación anterior:

$$
V=100 /\left[i+\exp \left(a+b \cdot S^{2} \cdot \exp (H)\right]\right.
$$

De igual forma es posible obtener una expresión para calcular el período de almacenamiento $\underline{\mathrm{S}}$, para valores dados de $\underline{\mathrm{V}}$ y $\underline{\mathrm{H}}$ :

$$
\mathrm{S}=[(\mathrm{Lv}-\mathrm{a}) / \mathrm{b} \cdot \exp (\mathrm{H})]
$$

\section{Ecuación de Contenido de Humedad en Equilibrio}

Los datos observados de T y HR del aire, y de $\mathrm{H}$ de las semillas se utilizaron para verificar si la ecuación de Roa (Rossi y Roa, 1980), para determinar CHE de las semillas de frijol se ajustaba a los valores encontrados para la variedad Calima. La ecuación propuesta es la siguiente:

$$
\begin{aligned}
& \mathrm{CHE}=\mathrm{A} \cdot \exp (\mathrm{B}) \\
& \text { donde: } \mathrm{A}=\mathrm{a} \cdot \mathrm{R}+\mathrm{b} \cdot \mathrm{R}^{2}+\mathrm{c} \cdot \mathrm{R}^{3} \\
& \mathrm{y}: \mathrm{B}=\left(\mathrm{d} \cdot \mathrm{R}+\mathrm{e} \cdot \mathrm{R}^{2}+\mathrm{f} \cdot \mathrm{R}^{3}+\mathrm{g} \cdot \mathrm{R}^{3}+\mathrm{g} \cdot \mathrm{R}^{4}\right)(\mathrm{h}+\mathrm{T})
\end{aligned}
$$

donde CHE es el contenido de humedad en equilibrio de las semillas en base seca (bs, decimal); $\underline{\mathrm{R}}$ es la HR del aire (decimal); $\mathrm{T}$ es la tempera $\left({ }^{\circ} \mathrm{C}\right)$ del aire en contacto con las semillas; exp el operador exponencial; $\mathrm{y} \underline{\mathrm{a}}, \underline{\mathrm{b}}, \underline{\mathrm{c}}, \underline{\mathrm{d}}, \underline{\mathrm{e}}, \underline{\mathrm{f}}, \mathrm{g}$, son constantes que para el caso de semillas de frijol son iguales a:

$\mathrm{a}=0.892910 \mathrm{~b}=0.636490 \mathrm{e}=1.092500 \mathrm{~d}=0.02210$

$\mathrm{e}=0.039437 \mathrm{f}=0.035660 \mathrm{~g}=-0.017932 \mathrm{~h}=273.0$

Para convertir los datos de CIHE obtenidos con esta ecuación de bs decimal a bh porcentaje, es necesario utilizar la siguiente relación:

$$
H=100 . S /(1+S)
$$

donde $\mathrm{H}$ es la humedad de la semilla (\% bh) y S es la humedad de la semilla (bs, decimal).

\section{RESULTADOS Y DISCUSIÓN}

\section{A. Calidad de la Semilla}

La calidad de las semillas se evaluó antes y después del período de almacenamiento, tal como se describió en la metodología. En el Cuadro 1 se han resumido algunos de los resultados obtenidos, luego de evaluar la calidad física y fisiológica de las diferentes muestras de semillas guardadas en AH. Aunque las evaluaciones fueron hechas cada dos semanas, durante 
Cuadro 1. Datos experimentales de humedad $(\mathrm{H})$, germinación $(\mathrm{G})$ y emergencia(E) de semillas de frijol, variedad Calima, para los siete niveles de humedad, durante las 32 semanas (PA) de AFI a $32^{\circ} \mathrm{C}$

\begin{tabular}{|c|c|c|c|c|c|c|c|}
\hline$\underset{(\text { sem) }}{\text { PA }}$ & $\underset{(\%)}{\mathbf{H}}$ & $\underset{(\%)}{\mathbf{G}}$ & $\underset{(\%)}{\mathrm{E}}$ & $\underset{(\text { sem) }}{\text { PA }}$ & $\underset{(\%)}{\mathbf{H}}$ & $\underset{(\%)}{\mathbf{G}}$ & $\underset{(\%)}{E}$ \\
\hline 0 & 10.2 & 96 & 90 & 0 & 12.7 & 96 & 86 \\
\hline 4 & 10.2 & 95 & 86 & 4 & 12.6 & 93 & 86 \\
\hline 8 & 10.3 & 95 & 86 & 8 & 12.5 & 92 & 85 \\
\hline 12 & 10.3 & 96 & 86 & 12 & 12.4 & 93 & 84 \\
\hline 16 & 10.4 & 96 & 85 & 16 & 12.3 & 90 & 83 \\
\hline 20 & 10.5 & 92 & 85 & 20 & 12.1 & 93 & 79 \\
\hline 24 & 10.2 & 92 & 86 & 24 & 12.2 & 83 & 75 \\
\hline 28 & 10.3 & 93 & 85 & 28 & 12.1 & 78 & 53 \\
\hline 32 & 10.5 & 92 & 86 & 32 & 12.3 & 65 & 38 \\
\hline 0 & 10.9 & 98 & 89 & 0 & 13.0 & 94 & 84 \\
\hline 4 & 11.0 & 95 & 86 & 4 & 12.8 & 93 & 86 \\
\hline 8 & 11.1 & 95 & 87 & 8 & 12.8 & 92 & 85 \\
\hline 12 & 10.8 & 93 & 87 & 12 & 13.0 & 89 & 83 \\
\hline 16 & 11.0 & 93 & 85 & 46 & 12,7 & 88 & 82 \\
\hline 20 & 11.3 & 93 & 85 & 20 & 12.8 & 84 & \\
\hline 24 & 11.0 & 92 & 86 & 24 & 12.8 & 76 & 69 \\
\hline 28 & 11.0 & 92 & 82 & 28 & 12.9 & 65 & 42 \\
\hline 32 & 11.0 & 90 & 79 & 32 & 12.7 & 54 & 29 \\
\hline 0 & 11.4 & 98 & 88 & 0 & 14.3 & 96 & 83 \\
\hline 4 & 11.3 & 95 & 87 & 4 & 13.9 & 94 & 83 \\
\hline 8 & 11.4 & 94 & 86 & 8 & 14.3 & 89 & so \\
\hline 12 & 10.9 & 94 & 84 & 12 & 13.7 & 90 & 73 \\
\hline 16 & 11.5 & 93 & 83 & 16 & 13.7 & 76 & 67 \\
\hline 20 & 11.4 & 91 & 83 & 20 & 14,0 & 56 & 45 \\
\hline 24 & 11.3 & 90 & 82 & 24 & 13.9 & 33 & 15 \\
\hline 28 & 11.3 & 91 & 80 & 28 & 13.8 & 0 & 0 \\
\hline 32 & 11.4 & 88 & 75 & 32 & 13.7 & 0 & 0 \\
\hline 0 & 12.1 & 94 & 88 & & & & \\
\hline 4 & 12.2 & 94 & 87 & & & & \\
\hline 8 & 12.2 & 91 & 87 & & & & \\
\hline 12 & 11.8 & 90 & 85 & & & & \\
\hline 16 & 12.3 & 90 & 83 & & & & \\
\hline 20 & 12.2 & 91 & 84 & & & & \\
\hline 24 & 12.0 & 88 & so & & & & \\
\hline 28 & 12.1 & 88 & 79 & & & & \\
\hline 32 & 12.2 & 80 & 69 & & & & \\
\hline
\end{tabular}

ocho meses, sólo se presentan resultados mensuales, con el fin de resumir la información y simplificar la interpretación. El cuadro presenta, para cada uno de los siete niveles de $\mathrm{H}$, el período de almacenamiento, el contenido de $\mathrm{H}$ al final de ese período, y la germinación y emergencia de las semillas.

El peso de cada una de las muestras de los diferentes tratamientos antes y después del período de $\mathrm{AH}$, aunque no están reportados, sí fueron determinados. Se encontró que el peso inicial que en promedio fue de 950 g (800 g semilla y 150 g M recipiente), presentó disminuciones para cada una de las muestras con un coeficiente de variación máximo de $0.12 \%$; lo que equivale a una diferencia de peso de menos de $1.2 \mathrm{~g}$. Estos resultados, junto con los datos de humedad, permiten concluir que los recipientes mantuvieron su hermeticidad durante el período de almacenamiento y que la HR del aire exterior no afectó la $\mathrm{H}$ de las semillas.

\section{B. Ecuación de viabilidad}

Los datos observados de germinación y emergencia a lo largo de las 32 semanas de almacenamiento presen$\tan \mathrm{h}$ forma sigmoidal característica reportada por varios investigadores (Roberts, 1961, 1972; Ellis, 1988).

Los valores de germinación y emergencia vs tiempo fueron sometidos a un análisis de regresión utilizando la transformación logit de la ecuación (1) y el modelo de la ecuaciones (2) y (3), tal como fueron descritas en la sección de Metodología. El análisis estadístico produjo los

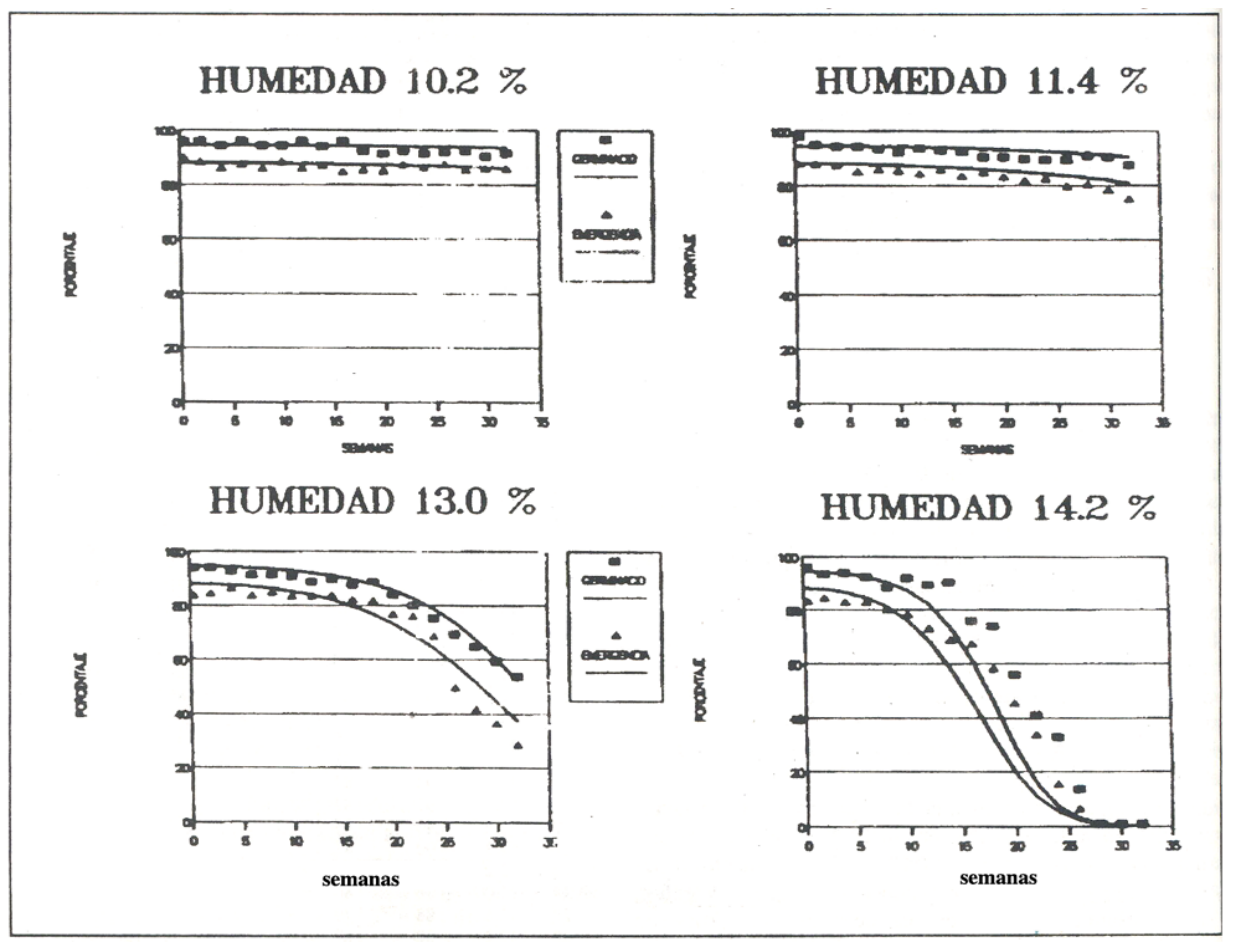

Figura 1. Datos experimentales y calculados de germinación y emergencia de semillas de frijol en $\mathrm{AH}$ a $30^{\circ} \mathrm{C}$. 
siguientes resultados:

\begin{tabular}{lll} 
& Germinación & Emergencia \\
\hline Coeficiente a: & -2.8811 & -2.0013 \\
Coeficiente b: & 6.6688 E-09 & 6.0260 E-09 \\
Correlación $r^{2}:$ & 0.854 & 0.926 \\
\hline
\end{tabular}

Utilizando la ecuación (3), es posible simular los datos de germinación y emergencia como función de la $\mathrm{H}$ de h semillas y del tiempo de almacenamiento. En la Figura 1 se presentan los datos observados y los calculados para cuatro niveles de $\mathrm{H}$ - Las Figuras 2 y 3 representan superficies de respuesta para germinación y emergencia, respectivamente, en función de la $\mathrm{H}$ de la semilla y del tiempo de almacenamiento. Se puede apreciar que la viabilidad disminuye a medida que aumenta la $\mathrm{H}$ de las semillas o el tiempo de almacenamiento; y que las semillas mantienen su viabilidad cuando se almacenan con $\mathrm{H}$ bajas durante cortos períodos de almacenamiento.

Para determinar el máximo, período de almacenamiento para semillas con un contenido de $\mathrm{H}$ dado; se utiliza la ecuación (4) derivada del modelo; de esta forma es posible calcular el período de $\mathrm{AH}$ permisible, dependiendo de la $\mathrm{H}$ de las semillas y de la viabilidad esperada al final del almacenamiento. En la Figura 4 se muestran los tiempos de $\mathrm{AH}$ permisibles para lotes de

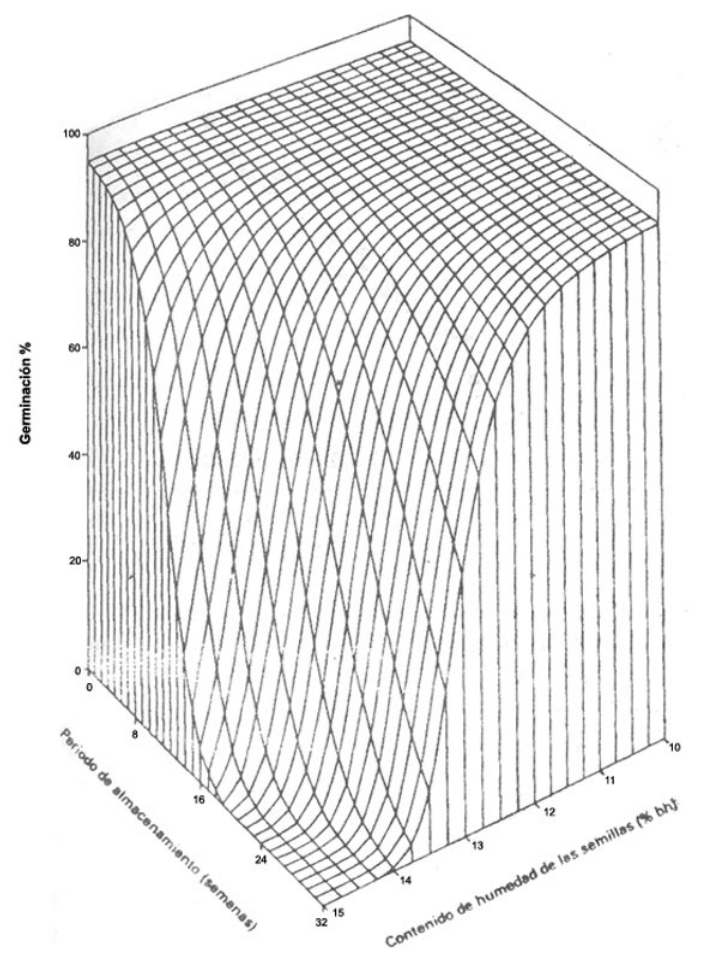

Figura 2. Porcentaje de germinación de semillas de frijol en función de su contenido de humedad y del período de almacenamiento hermético a $30^{\circ} \mathrm{C}$. semilla de frijol variedad Calima, con germinación inicial de $96 \%$ y emergencia inicial del $88 \%$, si se desea que la germinación final sea $90 \%$ o la emergencia final su $85 \%$, en un rango de $\mathrm{H}$ de 10 al $14 \%$ (bh). Se puede observar por ejemplo, que es posible mantener semillas de frijol en AH durante 32 semanas sin que la germinación final sea inferior al $90 \%$, siempre y cuando la semilla tenga una $\mathrm{H}$ máxima del $11.5 \%$. También se observa que si se almacena la semilla con $13 \%$ de H, podrá ser guardada en AH solamente durate 10 semanas, al final de las cuales las semillas tendrán un índice de emergencia del $85 \%$, o durante 16 semanas, al final de las cuales las semillas tendrán un $90 \%$ de germinación.

\section{Ecuación de Contenido de Humedad en Equilibrio}

Tal como se describió en la sección de Metodología, al final $\mathrm{M}$ período de almacenamiento se determinaron la $\mathrm{T}$ y HR del aire dentro del recipiente y la $\mathrm{H}$ de la semilla en equilibrio en esas condiciones, con el fin de compararlas con los datos obtenidos utilizando las ecuaciones (5), (6), (7) y (8), presentadas anteriormente. El Cuadro 2 presenta los datos experimentales de CHE, T y HR del aire y los valores de CITE calculados utilizando las ecuaciones mencionadas.

La Figura 5 muestra la comparación entre los valores medidos y calculados de CHE. Se puede observar

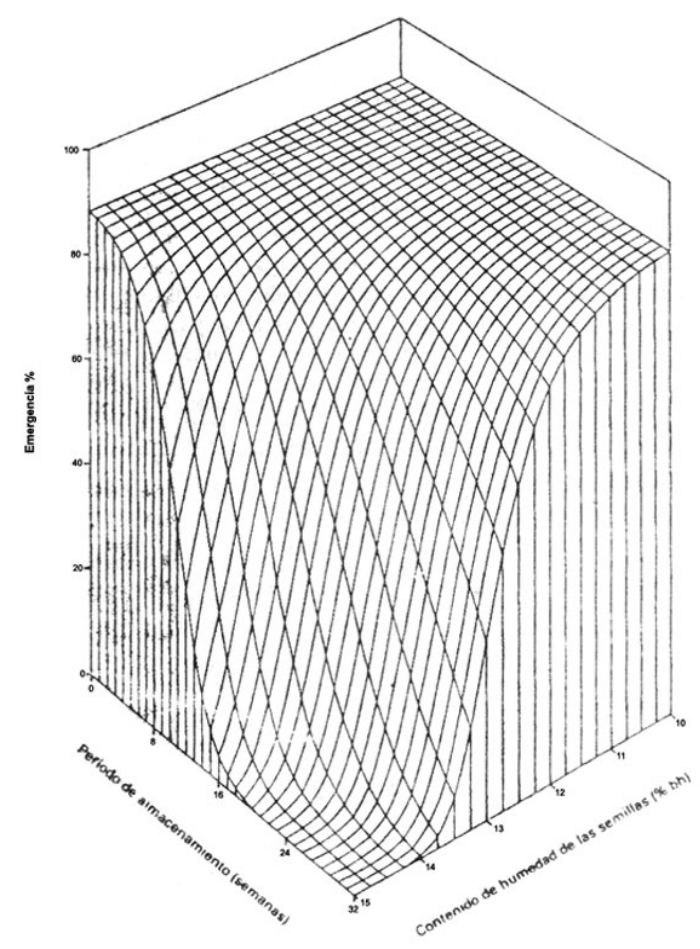

Figura 3. Porcentaje de emergencia de semillas de frijol en función de su contenido de humedad y del periodo de almacenamiento hermético a $30^{\circ} \mathrm{C}$. 
Cuadro 2. Contenido de humedad en equilibrio (CHE) de semillas de frijol. Datos experimentales (CHEe) y calculados (CHEc) en función de la temperatura M y humedad relativa (HR)

\begin{tabular}{cccccccc}
\hline $\begin{array}{c}\text { Temp. } \\
\left({ }^{\circ} \mathrm{C}\right)\end{array}$ & $\begin{array}{r}\text { HR } \\
\left({ }^{\circ} \mathrm{C}\right)\end{array}$ & $\begin{array}{r}\text { CHEe } \\
(\%)\end{array}$ & $\begin{array}{c}\text { CHEc } \\
(\%)\end{array}$ & $\begin{array}{c}\text { Temp. } \\
\left({ }^{\circ} \mathrm{C}\right)\end{array}$ & $\begin{array}{c}\text { HR } \\
\left({ }^{\circ} \mathrm{C}\right)\end{array}$ & $\begin{array}{r}\text { CHEe } \\
(\%)\end{array}$ & $\begin{array}{c}\text { CHEc } \\
(\%)\end{array}$ \\
\hline 29.8 & 47.8 & 10.1 & 10.4 & 28.6 & 58.8 & 12.1 & 12.0 \\
27.4 & 48.7 & 10.2 & 10.6 & 28.3 & 59.8 & 12.1 & 12.1 \\
29.5 & 48.0 & 10.3 & 10.4 & 22.9 & 59.9 & 12.1 & 12.4 \\
28.8 & 47.2 & 10.3 & 10.3 & 26.5 & 58.4 & 12.2 & 12.0 \\
26.6 & 49.1 & 10.4 & 10.7 & 26.4 & 58.0 & 12.2 & 11.9 \\
28.5 & 47.7 & 10.5 & 10.4 & 26.5 & 59.7 & 12.2 & 12.2 \\
26.3 & 48.2 & 10.5 & 10.6 & 27.8 & 57.9 & 12.2 & 11.9 \\
25.1 & 48.8 & 10.6 & 10.7 & 26.5 & 59.9 & 12.3 & 12.2 \\
29.5 & 52.4 & 10.9 & 11.0 & 26.2 & 59.0 & 12.3 & 12.1 \\
29.9 & 51.7 & 10.9 & 10.9 & 30.0 & 60.2 & 12.3 & 12.1 \\
28.5 & 52.1 & 11.0 & 11.0 & 26.1 & 60.2 & 12.4 & 12.3 \\
26.4 & 52.2 & 11.0 & 11.1 & 29.8 & 58.8 & 12.6 & 11.9 \\
26.7 & 52.8 & 11.0 & 11.2 & 26.4 & 61.4 & 12.7 & 12.5 \\
27.0 & 52.1 & 11.0 & 11.0 & 26.1 & 61,6 & 12.8 & 12.6 \\
28.6 & 55.3 & 11.2 & 11.4 & 26.6 & 61.4 & 12.8 & 12.5 \\
27.8 & 54.1 & 11.3 & 11.3 & 27.7 & 61.0 & 12.9 & 12.4 \\
27.6 & 52,4 & 11,3 & 11.1 & 28.4 & 60.6 & 12.9 & 12.3 \\
31.1 & 53.9 & 11.3 & 11.1 & 26.3 & 61.5 & 13.0 & 12.5 \\
29.9 & 53.4 & 11.3 & 11.1 & 26.4 & 66.7 & 13.5 & 13.5 \\
25.4 & 52.9 & 11.4 & 11.2 & 27.4 & 66.6 & 13.6 & 13.4 \\
26.4 & 53.8 & 11.4 & 11.3 & 28.0 & 65.7 & 13.6 & 13.2 \\
28.5 & 54.2 & 11.4 & 11.3 & 26.8 & 66.4 & 13.7 & 13.4 \\
26.4 & 55.2 & 11.6 & 11.5 & 27.5 & 65.9 & 13.7 & 13.3 \\
30.0 & 57.5 & 12.0 & 11.7 & 28.5 & 65.1 & 13.8 & 13.1 \\
26.1 & 58.3 & 12.0 & 12.0 & 27.5 & 65.5 & 14.0 & 13.2 \\
28.4 & 57,6 & 12.1 & 11.8 & & & & \\
\hline & & & & & & &
\end{tabular}

que el modelo se ajusta bien para semillas de frijol variedad Calima, dentro de los rangos de $\mathrm{T}\left(23\right.$ a $\left.30^{\circ} \mathrm{C}\right)$ y $\mathrm{H}$ (10 a $14 \%$ bh) estudiados.

Con esta información es posible determinar las características termodinámicas del aire dentro de los recipientes. El Cuadro 3 presenta los valores de humedad de la semilla, temperatura de bulbo seco (Tbs), temperatura de bulbo húmedo (Tbh), humedad relativa (HR), presión del vapor de agua $(\mathrm{Pv})$ y déficit de presión de vapor (DPV) del aire, para cada uno de los siete niveles

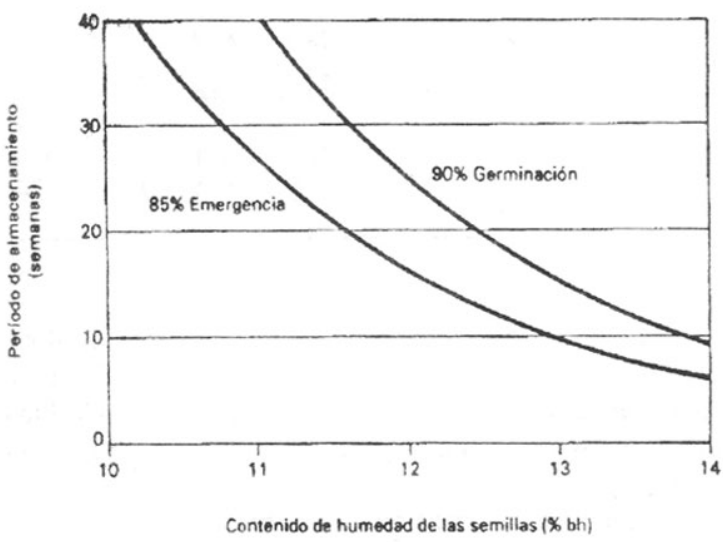

Figura 4. Periodo de almacenamiento hermético a $30^{\circ} \mathrm{C}$ y contenido de humedad de las semillas de frijol requeridos para mantener en $90 \%$ su germinación y en $85 \%$ su emergencia. de humedad estudiados.

La ecuación de CHE permite calcular cuáles son los valores de T y HR necesarios para que las semillas de frijol alcancen una $\mathrm{H}$ determinada. Estos valores se presentan en el Cuadro 4 y en la Figura 6.

Así por ejemplo, se puede observar que para que las semillas de frijol alcancen una $\mathrm{H}$ del $11 \%$ (bh), es necesario que el aire en contacto con ellas esté a $40^{\circ} \mathrm{C}$ y $56 \%$ $\mathrm{HR}$; o a $30^{\circ} \mathrm{C}$ y $53 \% \mathrm{HR}$; o a $20^{\circ} \mathrm{C}$ y $49 \% \mathrm{HR}$; o cualquier otra combinación de valores de T y HR definida por la línea de $\mathrm{H}$ del $11 \%$. Esta información es muy útil cuando no se dispone de otro método para la determinación de la $\mathrm{H}$ de las semillas ano de su almacenamiento.

\section{Aplicabilidad del AH para los Pequeños Agricultores}

Los resultados encontrados en este estudio permiten concluir que es posible adaptar la tecnología de $\mathrm{AH}$ a bajas temperaturas, bajos contenidos de humedad y largos períodos de almacenamiento a sistemas de $\mathrm{AH}$ a temperatura y humedades "altas", para la conservación de pequeñas cantidades de semillas de frijol de buena calidad inicial durante cortos períodos de tiempo.

El método, de $\mathrm{AH}$ a corto plazo es fácilmente aplicable, siempre y cuando la semilla de frijol que se va a únacenar sea de buena calidad, sea secada hasta las humedades recomendadas se haga un adecuado control de insectos antes del almacenamiento, se disponga de los empaques apropiados, y se almacene en lugares frescos y ventilados, donde la temperatura ambiente no sobrepase $\operatorname{los} 30^{\circ} \mathrm{C}$.

Cuadro 3. Propiedades termodinámicas del aire en el interior de recipientes, dependiendo del contenido de humedad de semillas

\begin{tabular}{cccccc}
\hline $\begin{array}{c}\text { Humedad } \\
(\% \mathbf{b h})\end{array}$ & $\begin{array}{c}\text { Tbs } \\
\left({ }^{\circ} \mathbf{C}\right)\end{array}$ & $\begin{array}{c}\mathbf{T B h} \\
\left({ }^{\circ} \mathbf{C}\right)\end{array}$ & $\begin{array}{c}\text { HR } \\
(\boldsymbol{\%})\end{array}$ & $\begin{array}{c}\text { Pv } \\
(\mathbf{m m} \mathbf{H g})\end{array}$ & $\begin{array}{c}\text { DP } \\
(\mathbf{m m} \mathbf{H g})\end{array}$ \\
\hline 10.2 & 30.0 & 21.4 & 46.6 & 15.0 & 16.8 \\
10.9 & 30.0 & 22.4 & 52.0 & 16.7 & 15.2 \\
11.4 & 30.0 & 23.0 & 55.5 & 17.6 & 14.2 \\
12.2 & 30.0 & 23.9 & 60.5 & 19.0 & 12.8 \\
12.7 & 30.0 & 24.4 & 63.5 & 20.2 & 11.6 \\
13.0 & 30.0 & 24.7 & 65.0 & 20.8 & 11.0 \\
14.3 & 30.0 & 25.7 & 71.0 & 22.9 & 9.0 \\
\hline
\end{tabular}

Cuadro 4. Humedad relativa del aire en equilibrio con semillas frijol a diferentes temperaturas

\begin{tabular}{ccccccc}
\hline $\begin{array}{c}\text { Temp. } \\
\left({ }^{\circ} \mathbf{C}\right)\end{array}$ & $\mathbf{1 0}$ & $\mathbf{1 1}$ & $\mathbf{1 2}$ & $\mathbf{1 3}$ & $\mathbf{1 4}$ & $\mathbf{1 5}$ \\
\hline 10 & 38.5 & 46.2 & 53.3 & 59.6 & 65.0 & 69.7 \\
20 & 41.7 & 49.5 & 56.5 & 62.4 & 67.5 & 71.9 \\
30 & 45.0 & 52.8 & 59.4 & 65.0 & 69.8 & 73.8 \\
40 & 48.4 & 55.9 & 62.2 & 67.4 & 71.8 & 75.5 \\
\hline
\end{tabular}




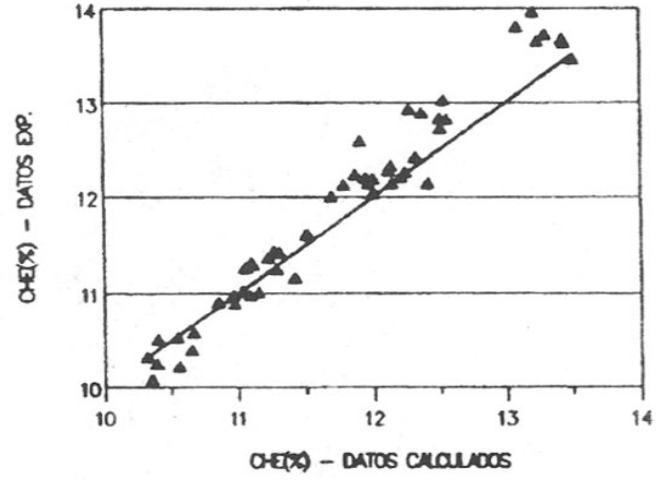

Figura 5. Comparación entre datos experimentales y calculados del contenido de humedad en equilibrio de semillas de frijol variedad Calima.

Uno de los problemas más graves relacionados con el secamiento de las semillas hasta un nivel de humedad apropiado para el AH, es la dificultad existente en la determinación de la $\mathrm{H}$ de las semillas, pues los métodos utilizados por los agricultores rara vez son precisos. Esta situación puede remediarse si se cuenta con instrumentos tan sencillos como el sicrómetro o un termómetro o higrómetro para medir la $\mathrm{T}$ y HR del aire de secado. Con estas mediciones y la información del Cuadro 4 o de la Figura 6, se puede encontrar el CHE de las semillas en esas condiciones.

También esencial en el AH es el control de insectos, especialmente gorgojos, antes del almacenamiento. Para lograrlo existen diferentes métodos, tal como fueron descritos anteriormente.

Otro punto importante en el AH es la selección del empaque adecuado, esta selección se debe hacer teniendo en cuenta: a) La cantidad de semillas que se van a almacenar; b) La debilidad y el precio del empaque; c) La facilidad del manejo y las condiciones del transporte y del almacenamiento; d) Las características de hermeticidad que impiden la de vapor de agua y a su vez el es-

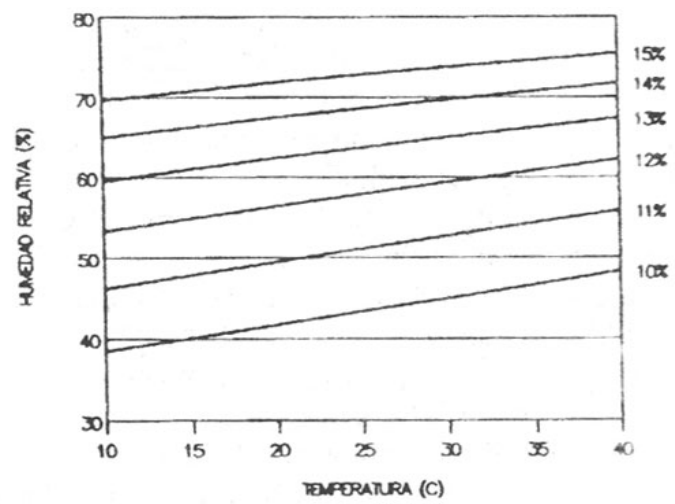

Figura 6. Contenido de humedad en equilibrio de semillas de frijol Calima en función de la temperatura y humedad relativa del aire. cape de insecticidas previamente aplicados a la semilla y que garanticen una barrera contra plagas y protección contra daño mecánico; y e) La facilidad de abrir el recipiente logrando cada vez un sellado hermético (Harrington y Douglas, 1970; Justice y Bass, 1978; Freire y Mumford,1986).

Los pequeños agricultores, con parcelas que en promedio tienen menos de 3 ha de extensión, sólo requieren cerca de $140 \mathrm{~kg}$ de semilla de frijol para la próxima siembra, cantidad ésta que ocupa un volumen de sólo $0.2 \mathrm{~m}^{3}$ (54 galones). Existe toda una serie de empaques metálicos y plásticos, baratos y de fácil consecución que pueden ser usados teniendo en cuenta las características anotadas anteriormente, en particular las referentes a la impermeabilidad y al método de cerramiento. Es importante que el recipiente sea llenado con semillas a su máxima capacidad, disminuyendo así la cantidad de aire que queda dentro del recipiente. Una vez almacenada la semilla, el empaque debe permanecer herméticamente cerrado hasta el momento en que se va a utilizar la semilla.

En este estudio se utilizó una temperatura de almacenamiento de $30^{\circ} \mathrm{C}$; sin embargo, en la mayoría de las regiones productoras de frijol a temperatura promedio es inferior a este valor. Si se considera que por cada $5^{\circ} \mathrm{C}$ que se disminuya la temperatura se duplica el potencial de almacenamiento de las semillas (Harrington, 1959), se puede concluir que el $\mathrm{AH}$ a temperaturas menores a $\operatorname{los} 30^{\circ} \mathrm{C}$ significará menor deterioro de la calidad de las semillas y un mayor tiempo de almacenamiento permisible.

Cabe repetir que el almacenamiento no mejora la calidad de las semillas y que las semillas de mala calidad no almacenan bien. En consecuencia, el agricultor debe preocuparse por obtener semillas de buena calidad, que son las que poseen el potencial necesario para sobrevivir durante el periodo de almacenamiento.

Siguiendo estas recomendaciones, el método descrito en este trabajo puede llegar a tener una amplia aplicabilidad entre los pequeños agricultores productores de frijol, permitiéndoles mantener la calidad de sus semillas durante el tiempo necesario.

\section{CONCLUSIONES}

De la información presentada se puede concluir lo siguiente:

1. Semillas de frijol variedad Calima, provenientes de un lote con buena calidad inicial (germinación $96 \%$ y emergencia $88 \%$, almacenadas en empaques herméticos en un ambiente a $30^{\circ} \mathrm{C}$ mantuvieron su calidad hasta por 32 semanas al ser almacenadas con contenidos de humedad inferiores al 12\% (bh).

2. Es posible predecir variaciones en los índices de germinación y emergencia de las semillas de frijol variedad Calima de buena calidad almacenadas por 
corto tiempo en empaques herméticos en un ambiente de 30 'C en un rango de humedad del 10 al $14 \%$ (bh), mediante la utilización de la ecuación (3) presentada en los resultados.

3. Es posible calcular el contenido de humedad de las semillas de frijol variedad Calima, en equilibrio con el aire, como función de la T y la HR del aire que las rodea, mediante la utilización de la ecuación que se presentó en los resultados.

\section{BIBLIOGRAFÍA}

BASS, LN. 1973. Controlled atmosphere and seed storage. Seed Science \& Technology, 1:463462.

- - - - 1975. Seed moisture and storage. Seed Science \& Technology, 3:743-746.

BERGER, R.D. 1981. Comparison of the Gompertz and Logistic equations to describe plant disease progress. Phytopathology, 71:716-719.

BURRELL, N.J. 1990. Effect of air tight storage on insect pests of stored products. In Controlled Atmosphere Storage of Crains Elsevier Scientific Publishing Company. 601 p.

CIAT. 1988a. CIAT Report. 130 p.

1988b. Trends in CIAT commodities.

DELOCHE, J.C. 1973. Precepts of seed storage. Proceedings short course for seedsmen. Vol. 16. Seed Technology Lab. Mississippi State University.

- - - -1980 . Some thoughts on seed storage. Proceedings short course ofr seedsmen. Vol. 2. Seed Technology Lab. Mississippi State University,

- - - - 1985. Physiological seed quality. Proceedings short course for seedsmen. Vol. 27. Seed Technology Lab. Mississippi State University.

ELLIS, R.H. 1988. The viability equation. seed viability nomographs and practical advice on seed storage. Seed Science \& Technology, 16:29-50

- - - ROBERTS, F-H. 1980. Improved equations for the prediction of seed longevity. Annals of Botanny. 45:13-30.

FAO. 1987. Generación de tecnologías adecuadas al desarrollo rural. Serie desarrollo rural No. 4. Oficina Regional para América Latina y el Caribe.

FREIRE, M.S.; MUNFORD, P.M. 1986. The efficiency of a range of containers in maintaining seed viability during storage. Seed Science \& Technology, 14:371-381.

HARRINGTON, J.F. 1959. Drying, storing and packaging seeds to maintain germination and vigor. Proceedings shortcourse for seedsmen, 89-107. Seed Technology Lab. Mississippi State University.

- 1973. Packaging seed for storage and shipment. Seed Science \& Technology, 1:478-492.

DOUGLAS, J.E. 1970. Seed storage and packaging. Applications for India. National Seeds Company lid. New Delhi. 222 p.

HARTY, R.L. 1977. The influence of storage conditions on bean seed quality. Queensland Agricultural Journal, 103:534-535.

HOUSTON, D.F. 1973. Linear function for comparing viability loss met in stored seeds. Seed Science \& Technology, 1:795-798.

ICA. 1987. Utilización de semilla certificada en Colombia. Semillas, ACOSEMILLAS, Bogotá Colombia, XII (3):28.

ISTA. 1985. International rules for seed testing. Seed Science \& Technology, 13:130-142.

JUSTICE, O.L., BASS, I-N. 1978. Principles and practices of seed storage USDA, Agriculture handbook No. 506. US Goverment Printing Office. Washington D.C. 289 p.

MARTINE7, J.B. 1986. Resultados preliminares de los ensayos \& almacenamiento de frijol en Centro Loja. Memorias III Mesa Redonda Latinoamericana sobre prevención de pérdidas poscosecha de granos. Colombia.

NAKAMURA, S. 1975. The most appropiate moisture content of seeds for their long life span. Seed Science \& Technology, S;

POPMGIS, F. 1974. Fisiología de sementes. AGIPLAN, Braisilia, Brasil.

ROBERTS, E.H. 1961. The viability of rice seed ej relation to temperature moisture content and gaseous environment. Annals of Botanny, 25:381-390.

— $-\ldots$ 1972. Viability of seeds. Syracuse University Press. Syracuse J 448 p.

ROOS, E.E. 1988. Phaseolus seed storage methodologies. lot P. Gep (ED.): Genetic resources of Phaseolus Beans. Kluwer Academi Publishers. London. 613 p.

ROSSI, SJ.; ROA, G. 1980. Secagem e armazenamento de produm agropecuarios con uso de energía solar e ar natural. Publicacao ACIESP No. 22. Sao Pablo, Brasil. 295 p.

SCHAWARTZ, H.H.; GALVE4 G.E. 1980. Bean production problem CIAT. Colombia. 300 p.

SIGAUT, F. 1980. Significance of underground storage in tradition systems of grain production.. In Controlled atmosphere storage of grains. Elsevier Scientific Publishing Company. 601 p.

WILSON, D.O. 1986. Threshing injury and mathematical modeling of storage deterioration in field bean seed (Phaseolus vulgaris L) Dissertation, Ohio State University. 